\title{
Sensor-Landmark Motion Planning in Mobile Robots
}

\author{
Abed C. Malti, Florent Lamiraux and Michel Taïx \\ LAAS/CNRS \\ 7, av. du Colonel Roche \\ F-31077 Toulouse Cedex 4 - France \\ first-name.lastname@laas.fr
}

\begin{abstract}
The robust execution of a planned trajectory is a critical issue in robotics. In this paper, we set the formal basis for a sensor-landmark-based motion planning approach. Our approach deals with inaccuracy of the map of the environment by producing motion features composed of a reference trajectory and of a set of pairs sensorlandmark over which the robot will control its motion. These motion features define along the trajectory closed-loop motion strategies for the robot. This approach enables us to produce secure motions by putting emphasis on landmarks that can represent a danger of collision. Our contribution is to state the problem in a generic formalism and to propose some simulations that show the relevance of our resolution approach.
\end{abstract}

\section{INTRODUCTION}

Mobile robot navigation is a difficult task for mainly three reasons. The first one comes from the high complexity of the path planning problem. The second one resides in the inaccuracy of the map of the environment used to plan the trajectory and the third reason is that the navigation task in cluttered environments requires precise localization. The first of these three issues has raised a lot of interest for the past fifteen years and solutions have been proposed to solve the path planning problem for simple or complex kinematic systems [4], [5]. To address the two last issues, the usual approach consists in localizing the robot along the trajectory with respect to local landmarks [2], [7], [9], [10], [13], while avoiding unexpected obstacle by reactive methods [1], [3], [6], [8]. Thus localization automatically corrects the imprecision of the map. Localizing the robot with respect to local landmarks like obstacles is obviously a good method since the position of the robot with respect to obstacles that are close to the robot is of greater importance than the position of the robot in a global coordinate frame. However, the localization task is usually indepedent from the trajectory following task and as a consequence, the landmarks chosen for navigation are not the best ones. Obstacles very close to the trajectory might not be chosen although they represent a high danger of collision. For a mobile robot of small size in large corridors, the choice of landmarks for navigation might not be very important, but for big and kinematically complex multi body robots moving close to obstacles, this choice is critical.

In this paper, we introduce a generic approach to include the choice of landmarks and sensors along a planned trajectory. The idea is to plan sensor-landmark primitive to perform sensor based motion along a trajectory. Instead of planning a trajectory in a first step and follow this trajectory in a second step, we will produce a sequence of sensorlandmark based motions. Two steps are necessary to reach this goal :

- One is a formal definition of sensor-landmark based motion. In our formalism we assume as known; the model of the environment, the non-collision planned trajectory and the sensors perceptions. The sensorlandmark planned motion is composed of a planned trajectory along which continuous pre-selected lists of sensor-landmark pairs are assigned. In the real world the robot uses the sensor-landmark planned motion to navigate and correct its trajectory. This paper is focus on how this correction will be done.

- The other step is to define sensor-landmark strategy selection to best fit the real world according to the reference trajectory. This step is future work.

We think that our approach can be applied in a more general context. For instance in manipulation, algorithms exist today to plan the motion of a mobile manipulator that will grasp an object, carry the object and put it at a specified place [11]. To execute such motions, we need to specify along each part of the motion which sensors and which landmarks should be taken into account. In the grasping step, the relative position of the end-effector with respect to the object is obviously the most relevant piece of information. This piece of information will be presented in a landmark-based motion defined later in this paper.

In Section II, we introduce the notation and definitions upon which we build our generic framework. The main output of this framework is a formal definition of a landmark-based motion. In Section III, we describe the main components of the software we are developping to reach the landmark-based motion planner we are aiming at. Finally, in Section IV, we provide the reader with a few simulation results that show the relevance of our approach.

\section{NotATion AND DEFINITION}

Let us consider an unspecified robot. $\mathbb{S}=\left\{s_{1}, \ldots, s_{n}\right\}$ is a set of $n$ sensors attached to the different bodies of this robot. $\mathbb{L}=\left\{l_{1}, l_{2}, \cdots l_{P}\right\}$ is the set of $P$ landmarks located in the workspace of the robot. In order to simplify 
the notations, $s_{i}$ and $l_{j}$ respectively will represent the configuration of the sensor $i$ and the landmark $j$ respectively in the workspace.

\section{A. The Sensor Image Function}

If $l_{j} \in \mathbb{L}$ is a visible landmark from a sensor $s_{i} \in \mathbb{S}$. The image of $l_{j}$ given by $s_{i}$ is defined as :

$$
\begin{array}{cccc}
\mathrm{T}_{j}^{i}: & \mathbb{L} \times \mathbb{S} \\
\left(l_{j}, s_{i}\right) & \longmapsto & \mathbb{R}^{v} \\
& \longmapsto & y_{j}^{i}=\mathrm{T}_{j}^{i}\left(l_{j}, s_{i}\right)
\end{array}
$$

As shown in this definition, the space mapped by $\mathrm{T}$ is an euclidian space of dimension $v$. The function $\mathrm{T}_{j}^{i}$ doesn't depend only on the kind of the sensor but on the kind of the landmark too. For example, the image functions for a camera and for a laser sensor in relation to a segment are not the same. As well as the image functions for a point and for a segment captured by a laser sensor are not the same.

\section{B. Kinematic Relation Between The Robot And Its Sensors}

Since the sensor $s_{i}$ is linked to the robot, there exists a kinematic relation that links the configuration $q \in \mathcal{C}$ of the robot to the sensor configuration $s_{i} \in \mathcal{C}$ :

$$
\begin{array}{rlc}
\Gamma_{i}: \mathcal{C} & \longrightarrow & \mathbb{S} \\
q & \longmapsto & s_{i}=\Gamma_{i}(q)
\end{array}
$$

The composition $(\mathrm{T} \circ \Gamma)$ of the two functions defined above gives directly the image of a landmark $l_{j}$ viewed by a sensor $s_{i}$ given the configuration of the robot :

$$
\begin{aligned}
& \psi_{j}^{i}: \mathbb{L} \times \mathcal{C} \quad \longrightarrow \quad \mathbb{R}^{m} \\
& \left(l_{j}, q\right) \longmapsto y_{j}^{i}=\psi_{j}^{i}\left(l_{j}, q\right)
\end{aligned}
$$

The ability of sensing a landmark by a sensor gives a sort of sensor-landmark association depending on the kind of the sensor and the kind of the sensed landmark. If we associate $L=\left(l_{1}, \ldots, l_{p}\right)^{t}, p$ landmarks from the workspace with the robot's sensors. By assuming that each landmark is mapped by only one sensor, we obtain $p$ pairs sensorlandmark $S-L=\left\{\left(s_{i}, l_{1}\right),\left(s_{j}, l_{2}\right), \ldots,\left(s_{k}, l_{p}\right)\right\}$. Then the next extended representation is obtained :

$$
\left(\begin{array}{c}
y_{1}^{i} \\
y_{2}^{j} \\
\cdots \\
y_{p}^{k}
\end{array}\right)=\left(\begin{array}{c}
\psi_{1}^{i}\left(l_{1}, q\right) \\
\psi_{2}^{j}\left(l_{2}, q\right) \\
\ldots \\
\psi_{p}^{k}\left(l_{p}, q\right)
\end{array}\right) ; i, j, k \in[1, n]
$$

For the sake of simplicity we use the notation defined below :

$$
Y=\Psi(L, q)
$$

\section{Related Spaces}

Our reasoning is based on two different spaces. The first one is related to a reference map: the model. In this model, we have a non-collision planned trajectory. At each robot configuration, we assume to be able to get the visible landmarks from the robot's sensors and then assess them images. The motion planing phase is processed in this space. It takes the planned trajectory as reference and transforms it in a sequence of sensorlandmark association. The planned motion is composed of a planned trajectory and a succession of sensor-landmark pairs. All entity (robot, sensor, landmark) located in that space is pointed out with an exponent $m$. For example $Y^{m}=\Psi\left(L^{m}, q^{m}\right)$ indicates the images of the visible map's landmarks viewed by sensors in the map's robot configuration $q^{m}$.

The second space is related to the real environment. In this space the planned motion is executed. The objects belonging to this space are pointed out with an exponent $r$. For example $Y^{r}=\Psi\left(L^{r}, q^{r}\right)$ indicates the real images of the visible real landmarks when the robot is in the real configuration $q^{r}$.

We assume there is no means to estimate neither $q^{r}$ nor $L^{r}$. Whereas $Y^{r}$ is available since it comes from the sensors measures.

One of the generic items our apprroach deals with, is when $L^{r} \neq L^{m}$. This assumption means that the configuration of the real landmarks in relation to global coordinate frame of the real space is different of the configuration of the map landmarks in relation to global coordinate frame of the map space. However, we assume that $L^{r}$ is in the neighbourhood of $L^{m}$.

Given these assumptions, next we define two main configurations of the robot that show where it has to be in the real environment to satisfy the planned motion.

\section{Localization}

Let us consider $q_{0}^{m}$ as a desired configuration of the robot. This configuration was carried out by assuming a perfect model of the robot and a well specified reference map of the workspace. In this reference configuration, the robot's sensors pick up $p$ landmarks $L^{m}=\left(l_{1}^{m}, \ldots, l_{p}^{m}\right)^{t}$ from the map. In this way, at the configuration $q_{0}^{m}, p$ sensor-landmark pairs are associated. $Y_{0}^{m}=\Psi\left(L^{m}, q_{0}^{m}\right)$ is the refrence image of the selected sensor-landmark pairs. Let us consider $q_{e}^{r}$ as the configuration of executing $q_{0}^{m}$ in the real space. $L^{r}=\left(l_{1}{ }^{r}, \ldots, l_{p}{ }^{r}\right)^{t}$ is the vector of the real landmarks located in this space and corresponding to $L^{m}$. $Y_{e}^{r}=\Psi\left(L^{r}, q_{e}^{r}\right)$ is a vector of dimension $v \times p$ representing the real image of the selected sensor-landmark pairs.

Definition 1: A configuration of localization $q_{l o c}^{m}$ (configuration related to the map space) is defined by the following equation called the localization equation :

$$
Y_{e}^{r}=\Psi\left(L^{m}, q_{l o c}^{m}\right)
$$

1) Resolution of the localization equation: (1) is obviously a non linear equation. By assuming $q_{l o c}^{m}$ in the neighborhood of $q_{0}^{m}$, the development of Taylor of this function at the first order is given by :

$Y_{e}^{r}=\Psi\left(L^{m}, q_{0}^{m}\right)+\left(\frac{\partial \Psi}{\partial q}\right)_{\left(L^{m}, q_{0}^{m}\right)}\left(q_{l o c}^{m}-q_{0}^{m}\right)+o\left(q_{l o c}^{m}-q_{0}^{m}\right)$

By adopting the notation $Y_{0}^{m}=\Psi\left(L^{m}, q_{0}^{m}\right)$ and neglecting the residue of the linearization :

$$
Y_{e}^{r}-Y_{0}^{m}=\left(\frac{\partial \Psi}{\partial q}\right)_{\left(L^{m}, q_{0}^{m}\right)}\left(q_{l o c}^{m}-q_{0}^{m}\right)
$$


This equation is a linear system of $v \times p$ equations and $\operatorname{dim}(\mathcal{C})$ unknowns (the dimension of the configuration space of the robot $\mathcal{C}$ ). Intuitevely, in the general case of several landmarks, it's impossible to find a $q_{l o c}^{m}$ that allows to satisfy the measured images in relation to the map space. Then, at the localized configuration, we try to put the robot in the map space such that it best fit the measured images $Y_{e}^{r}$. To do that, we minimize the error defined below according to least square criterion :

$$
J_{L S}=\left\|\left(Y_{e}^{r}-Y_{0}^{m}\right)-\left(\frac{\partial \Psi}{\partial q}\right)_{\left(L^{m}, q_{0}^{m}\right)}\left(q_{l o c}^{m}-q_{0}^{m}\right)\right\|
$$

Then the best fitting solution is given by :

$$
q_{l o c}^{m}-q_{0}^{m}=\left(\frac{\partial \Psi}{\partial q}\right)_{\left(L^{m}, q_{0}^{m}\right)}^{+}\left(Y_{e}^{r}-Y_{0}^{m}\right)
$$

(4) shows that $q_{l o c}^{m}$ depends on $Y_{0}^{m}$ and $Y_{e}^{r}$. Thereby we can write it as a function with respect to these variables :

$q_{l o c}^{m}\left(Y_{0}^{m}, Y_{e}^{r}\right)-q_{0}^{m}=\left(\frac{\partial \Psi}{\partial q}\right)_{\left(L^{m}, q_{0}^{m}\right)}^{+}\left(Y_{e}^{r}-\Psi\left(L^{m}, q_{0}^{m}\right)\right)$

Please Note that the solution is given as a difference and not as an absolute value. This difference is called the localization error. The configurations $q_{0}^{m}$ and $q_{l o c}^{m}$ belong to the configuration space related to the map space.

Now, let us see an exemple, that explains how the robot behaves while the localization. Figures 1, 2 and 3 show and explain qualitatively the different steps leading to the localization. As an indication, the localization equation suited to this example has the following expression :

$$
\left(\begin{array}{c}
\left(d_{1}^{2}\right)_{e}^{r} \\
\left(d_{2}^{1}\right)_{e}^{r}
\end{array}\right)=\left(\begin{array}{c}
\psi_{1}^{2}\left(l_{1}^{m}, q_{0}^{m}\right) \\
\psi_{2}^{1}\left(l_{1}^{m}, q_{0}^{m}\right)
\end{array}\right)
$$

Where $d$ : the right distance from the sensor to the associated landmark.

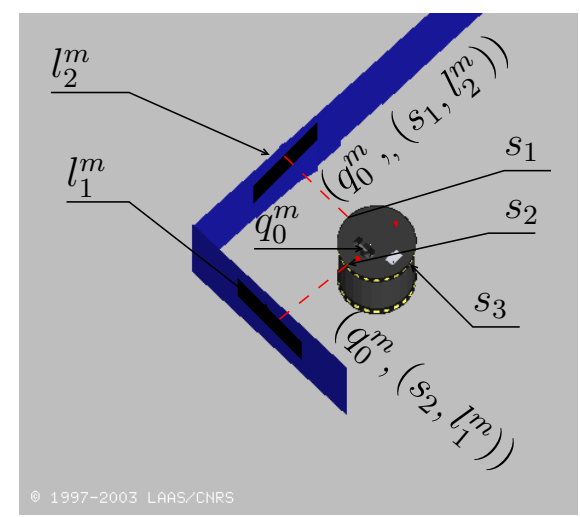

Fig. 1. The robot is at the desired configuration $q_{0}^{m}$ in the reference map. $s_{1}, s_{2}$ and $s_{3}$ are three UltraSonic sensors carried by the robot. $l_{1}^{m}$ and $l_{2}^{m}$ are the reference landmarks (assumed to be two segments) extracted from the walls. they are respectively associated to the sensor's $s_{2}$ and $s_{1} . s_{3}$ is not associated to any landmarks. The image of a pair sensor-landmark is assumed to be the right distance from the sensor to the direction of the segment.

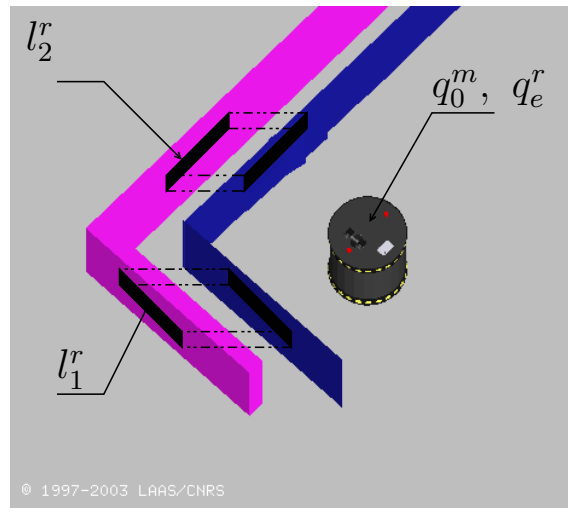

Fig. 2. The map space and the real space (with light color) are superposed. We can see that the reference map is not accurate $\left(l_{2}^{r} \neq l_{2}^{m}\right.$ and $\left.l_{1}^{r} \neq l_{1}^{m}\right)$. The real landmarks $l_{1}^{r}$ and $l_{2}^{r}$ are behind the references one $l_{1}^{m}$ and $l_{2}^{m}$ in relation to the robot. Whereas the real robot configuration $q_{e}^{r}$ corresponds to the desired one $q_{0}^{m}$

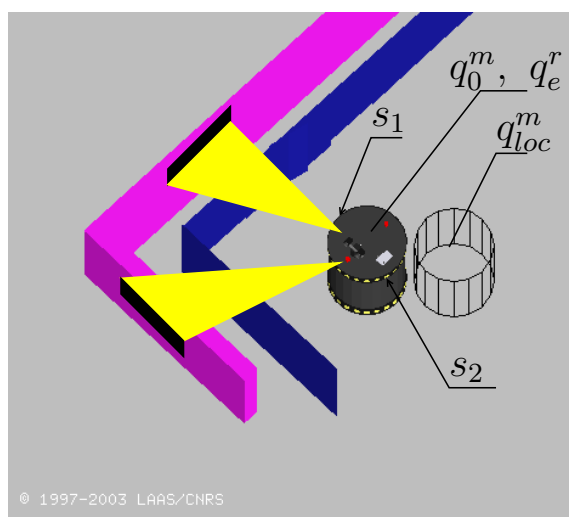

Fig. 3. The reference map and the real environment (with light color) are superposed. When it localizes itself in $q_{l o c}^{m}$, the robot believes that it is located behind the desired robot $q_{0}^{m}$ in the map space. Although it's not true in the real space. This happen because it senses that the landmarks are further and it assumes that the reference map is accurate.

\section{E. Correction}

The idea is to find a configuration of the robot in the real space such that when the localization is performed it believes it reaches $q_{0}^{m}$.

Definition 2: A map reference configuration $q_{M a p R e f}^{r}$ (related to the real environment) is defined as :

$$
q_{l o c}^{m}\left(Y_{0}^{m}, \Psi\left(q_{\text {MapRef }}^{r}, L^{r}\right)\right)-q_{0}^{m}=0
$$

This expression means that once the real robot in $q_{\text {MapRef }}^{r}$ and after performing a localization, it believes it reaches the desired configuration $q_{0}^{m}$ (the localization error is null).

1) Solving: (5) and (6) implies :

$$
\left(\frac{\partial \Psi}{\partial q}\right)_{\left(L^{m}, q_{0}^{m}\right)}^{+}\left(\Psi\left(L^{r}, q_{\text {MapRef }}^{r}\right)-\Psi\left(L^{m}, q_{0}^{m}\right)\right)=0
$$

We assume that $q_{\text {MapRef }}^{r}$ and $L^{r}$ respectively are in the neighborhood of $q_{0}^{m}$, the desired robot configuration, and 
$L^{m}$ respectively.

$$
\begin{gathered}
\Rightarrow\left(\frac{\partial \Psi}{\partial q}\right)_{\left(L^{m}, q_{0}^{m}\right)}^{+}\left(\left(\frac{\partial \Psi}{\partial q}\right)_{\left(L^{m}, q_{0}^{m}\right)}\left(q_{\text {MapRef }}^{r}-q_{0}^{m}\right)\right. \\
+\left(\frac{\partial \Psi}{\partial L}\right)_{\left(L^{m}, q_{0}^{m}\right)}\left(L^{r}-L^{m}\right) \\
\left.+o\left(L^{r}-L^{m}\right)+o\left(q_{\text {MapRef }}^{r}-q_{e}^{r}\right)\right)=0
\end{gathered}
$$

By neglecting the residues of the linearization in $L$ and in $q$ we obtain :

$$
\left(\frac{\partial \Psi}{\partial q}\right)_{\left(L^{m}, q_{0}^{m}\right)}^{+}\left(\frac{\partial \Psi}{\partial L}\right)_{\left(L^{m}, q_{0}^{m}\right)}\left(q_{\text {MapRef }}^{r}-q_{0}^{m}\right)+
$$

We assume that $q_{e}^{r}$ is in the neighbourhood of $q_{0}^{m}$. If we develop the expression of the measured image $Y_{e}^{r}=$ $\Psi\left(L^{r}, q_{e}^{r}\right)$ around $\left(L^{m}, q_{0}^{m}\right)$ and assume a null residue of linearization, we get :

$$
\begin{aligned}
\Psi\left(L^{r}, q_{e}^{r}\right)= & \Psi\left(L^{m}, q_{0}^{m}\right)+\left(\frac{\partial \Psi}{\partial q}\right)_{\left(L^{m}, q_{0}^{m}\right)}\left(q_{e}^{r}-q_{0}^{m}\right) \\
& +\left(\frac{\partial \Psi}{\partial L}\right)_{\left(L^{m}, q_{0}^{m}\right)}\left(L^{r}-L^{m}\right)
\end{aligned}
$$

Therefore :

$$
\begin{array}{r}
\left(q_{\text {MapRef }}^{r}-q_{0}^{m}\right)+\left(\frac{\partial \Psi}{\partial q}\right)_{\left(L^{m}, q_{0}^{m}\right)}^{+}\left(\Psi\left(L^{r}, q_{e}^{r}\right)-\Psi\left(L^{m}, q_{0}^{m}\right)\right. \\
\left.-\left(\frac{\partial \Psi}{\partial q}\right)_{\left(L^{m}, q_{0}^{m}\right)}\left(q_{e}^{r}-q_{0}^{m}\right)\right)=0
\end{array}
$$$$
\Rightarrow
$$$$
\begin{gathered}
q_{\text {MapRef }}^{r}\left(Y_{0}^{m}, Y_{e}^{r}\right)-q_{e}^{r}= \\
-\left(\frac{\partial \Psi}{\partial q}\right)_{\left(L^{m}, q_{0}^{m}\right)}^{+}\left(\Psi\left(L^{r}, q_{e}^{r}\right)-\Psi\left(L^{m}, q_{0}^{m}\right)\right)
\end{gathered}
$$

This solution is called the correction error. It gives a relative result with respect to the actual real robot configuration $q_{e}^{r}$. In this way, it will know the correction that it has to do to yield a null localization error. Like $q_{l o c}^{m}$, $q_{\text {MapRef }}^{r}$ depends of $Y_{e}^{r}$ and $Y_{0}^{m}$. The figure 4 represents the result of the correction on the robot configuration of the example presented before. The comparison of the robot in the desired map in 1 and in the real environment in 4 indicates that it seems reach its desired configuration in relation to the two moved walls (in terms of localization). Obviously this is not true in an absolute coordinate space. Even if the robot believes that it reaches the desired configuration, it cannot satisfy the reference images of all landmarks (see property2).

It is important to put the emphasis on the fact that the localization and correction processes presented here are subtly different from the classic one. We are not concerned about localizing the robot with respect to the global coordinate frame in the real world. Also, we take the geometric planned trajectory as a reference to best fit the real world. The concept of best fitting the real world is represented by a list of selected pairs sensor-landmark. The
Fig. 4. The desired robot and the references walls are represented with iron lines. The robot in the real space is moved by a rate of the correction error. If it performs a localization in the new configuration $q_{\text {MapRef }}^{r}$ it will find the iron robot configuration (a null localization error) and thus believes it satisfies the reference map.

strategy of selecting (planning) those primitives sensorlandmark is future work. However this concept will be introduced further in this paper by defining a sensorlandmark planned motion.

Property 1: The two equations (5) and (7) implies :

$$
q_{\text {MapRef }}^{r}\left(Y_{0}^{m}, Y_{e}^{r}\right)-q_{e}^{r}=-\left(q_{l o c}^{m}\left(Y_{0}^{m}, Y_{e}^{r}\right)-q_{0}^{m}\right)
$$

This property shows that as the robot localizes itself in relation to the desired configuration $q_{0}^{m}$ and the reference landmarks $L^{m}$, it simply has to carry out the inverse way in order to believe that it reaches $q_{0}^{m}$.

Property 2: $q_{\text {MapRef }}^{r}$ minimizes the sum of squares of euclidian distances between the reference images and the images of landmarks after the correction is carried out :

$$
D^{2}=\left\|\Psi\left(L^{r}, q_{\text {MapRef }}^{r}\right)-\Psi\left(q_{0}^{m}, L^{m}\right)\right\|^{2}
$$

In fact, this property express the residue of the solution given by the citerion $J_{L S}$.

\section{F. Sensor-Landmark Planned Motion}

Definition 3: A sensor-landmark planned motion is a continuous trajectory in the robot configuration space. At each configuration $q^{m}, L^{m}=\left\{l_{j}^{m}, \ldots, l_{j+p-1}^{m}\right\} p$ landmarks are selected and associated to the robot sensor's.

For a single sensor-landmark pair $\left(s_{i}, l_{j}\right)$ we define the sensor-landmark-based motion as :

$$
\begin{array}{rlc}
\mathrm{M}_{i j}: \mathcal{C} \times \mathbb{S} \times \mathbb{L} & \longrightarrow & \mathcal{C} \times \mathbb{S L} \\
\left(q, s_{i}, l_{j}\right) & \longmapsto & \left(q,\left(s_{i}, l_{j}\right)\right)
\end{array}
$$

For $p$ sensor-landmark pairs $S-L=$ $\left\{\left(s_{i}, l_{j}\right),\left(s_{i}, l_{j+1}\right), \ldots,\left(s_{k}, l_{j+p-1}\right)\right\} \quad$ we extend the above notation as :

$$
\begin{array}{rlc}
\mathrm{M}: \mathcal{C} \times \mathbb{S} \times \mathbb{L} & \longrightarrow & \mathcal{C} \times \mathbb{S} L \\
(q, S, L) & \longmapsto & (q,(S, L))
\end{array}
$$

\section{SOFTWARE INTEGRATION}

Our goal is planning sensor-landmark-based motion in object oriented platform built upon move3d [12]. The background architecture is spanned on four abstract classes representing the robot, the sensors, the landmarks and the sensor-landmark pairs. The abstract object class dedicated to the sensor-landmark pairs provides a straightforward representation of the image space. This one is very useful as a given sensor can be led to capture different sort of landmarks. In the opposite way a given landmark can be viewed by different kind of sensors. Note that if a derived sensor-landmark class exists, it necessary implies that the corresponding sensor and landmark classes already exist.

Up to now, we integrate a laser object class derived from the sensor abstract class. Both a $2 \mathrm{~d}$ point and a $2 \mathrm{~d}$ segment 
classes derived from the landmark abstract class. In this way, we must to generate two derived classes of sensorlandmark pair in the purpose of handling each kind of pair. The first one concerns the "LaserPoint2d" pair. It models a point viewed in a $2 \mathrm{~d}$ image space by laser sensor. The image function fitted to such a pair is given by :

$$
\begin{aligned}
\mathrm{T}: \mathbb{R}^{2} \times \mathbb{R}^{3} & \longrightarrow \mathbb{R}^{2} \\
\left(p_{j}, s_{i}\right) & \longmapsto Y
\end{aligned}
$$

With :

$$
Y=\left\{\begin{array}{l}
y_{x}=\left(x_{j}-x_{i}\right) \cos \left(\theta_{i}\right)+\left(y_{j}-y_{i}\right) \sin \left(\theta_{i}\right) \\
y_{y}=-\left(x_{j}-x_{i}\right) \sin \left(\theta_{i}\right)+\left(y_{j}-y_{i}\right) \cos \left(\theta_{i}\right)
\end{array}\right.
$$

Obviously this image is defined only if the point belongs to the sensing range of the laser. The second derived class represents the "LaserSegment2d" pairs. A segment is defined as a set of infinite number of aligned points bounded by tow ends points. Thus in $2 \mathrm{~d}$ workspace a segment is defined as a pair $\left(o^{1}, o^{2}\right)$ with $o^{1}\left(x^{1}, y^{1}\right)$ and $o^{2}\left(x^{2}, y^{2}\right)$. Since a segment is a non-oriented geometric entity, it's enough to define its orientation by an angle $\alpha_{0}=\left(\overrightarrow{\overrightarrow{o x}, o^{1} o^{2}}\right)$ belonging to the manifold $\left[-\frac{\pi}{2}, \frac{\pi}{2}\right]$. The image space is of dimension two. It is defined as the right distance projected from the center of the laser upon the direction of the segment. The second dimension of the space image is an angular one. It is the oriented angle $\left(\vec{S}, o^{1} o^{2}\right)$ made up by the laser and the segment. For the same reason as before this angle belongs to $\left[-\frac{\pi}{2}, \frac{\pi}{2}\right]$. The formulas used to express these images components are :

$$
\begin{aligned}
\mathrm{T}:\left(\mathbb{R}^{2} \times \mathbb{R}^{2}\right) \times \mathbb{R}^{3} & \longrightarrow \mathbb{R}^{2} \\
\left(\left(o_{j}^{1}, o_{j}^{2}\right), s_{i}\right) & \longmapsto Y
\end{aligned}
$$

With :

$$
Y=\left\{\begin{array}{l}
y_{d}=\left|\left(x_{i}-x_{j}^{1}\right) \sin \left(\alpha_{0}\right)-\left(y_{i}-y_{j}^{1}\right) \cos \left(\alpha_{0}\right)\right| \\
y_{\alpha}=\alpha_{0}-\theta_{i} ; y_{\alpha} \in\left[-\frac{\pi}{2}, \frac{\pi}{2}\right]
\end{array}\right.
$$

These two object classes handle the weight that has to be assigned to a sensor-landmark pair. This Weight is actually computed in relation to the distance between the sensor and its associated landmark. This choice enables to ensure the main characteristic of a continuous weight function.

\section{Simulation Results}

The preliminary framework presented above is applied to the hilare-trailer non-holonomic mobile robot of four degree of freedom in a simulation context. A laser sick sensor is mounted on the forward part of the robot. A second similar sensor is carried by the trailer platform. The derived object class "HilareTrailer" uses robot data structure of move3d to represent kinematic parameters of the robot needed for example to compute the robot-sensor configuration transfer function defined in §II-B. A libraray named "segkit", developped in our laboratory, is used to simulate the laser sensors of the robot.The figures 5 show the simulation results. The right side of the subfigures show an enlarge view of the related subfigure around a configuration point of the trajectory.
In a given reference map representing a part located in our laboratory we plan a trajectory from a start point to a goal point with move3d. The figure 5(a) represents that trajectory. Along the planned trajectory, the reference landmarks are selected and associated to the robot sensor's for making up the sensor-landmark pairs. In a given planned configuration $q_{0}$, we use the functions of "segkit" to determine the visible parts of the map's landmarks. Those visible landmarks are associated to the corresponding laser sensors in the robot configuration $q_{0}^{m}$. After sampling the planned trajectory, this process is carried out for each sampled configuration. The planned motion is then obtained. The enlarge view in figure 5(a) represents a configuration point of the sensor-landmark planned motion trajectory. The kind of landmark used in this simulation process is only the $2 \mathrm{~d}$ segments as they are better recognized by the laser sensor than the $2 \mathrm{~d}$ points. The dashed lines show the association between the two sensors of the robot and the reference landmarks.

Up to here, we described the sensor-landmark planned motion presented in §II-F. The second step consists on assuming a real environment different from the reference map. When the map space is superposed upon the real space, it appears that $L^{r} \neq L^{m}$. In figure 5(b) The real environment is illustrated with light color. If the robot executes perfectly the planned trajectory i.e. at each time $q_{e}^{r}=q_{0}^{m}$, it clearly appears that the execution will not be reliable in relation to the planned trajectory in the map space, in addition, in this case it will lead the robot to a collision in the real space. This would be worse when $q_{e}^{r} \neq q_{0}^{m}$. The enlarge view in figure 5(b) shows the real location of the expected landmarks. In this case, the images of the real landmarks will be different from those of the reference one. Consequently, the localization error is not null. So a correction has to be performed with respect to the new situation of the environment. The matching process between $L^{m}$ and $L^{r}$ is enabled by the library "segkit". When in a planned motion configuration $q_{0}^{m}$ the list of landmark $L^{m}$ doesn't match entirely $L^{r}$, the unmatched real landmarks are not taken into account. The simulated motion is presented in fig 5(c). The reference map and the planned trajectory are drawn with iron wire. We observe that the corrected motion executed from the planned motion fits the real environment better than the single execution of the planned trajectory. The main advantage of our approach is to keep the planned configuration locally unchangeable with respect to the landmarks frames. As seen in 5(c), neither for the robot nor for the walls the executed trajectory is different from the planned one.

\section{CONClusion}

In this paper, we have proposed a generic approach to produce motion features composed of a reference trajectory and a set of sensor-landmark pairs. These motion features define along the planned trajectory closed-loop motion strategies for the robot. The contribution of this paper is to propose a generic framework for the definition of these closed-loop motion features and to give a few examples to 
show the relevance of the approach. Indeed, any sensor on any robot and any landmark can be taken into account in our framework.

When the real space is close to the model space the simulations results show that the robot can fit the real world according to the planned trajectory. The work we are developping extends the approach for larger difference between the two spaces.

The next step of this work will consist in developing generic strategy planning algorithms within a software platform to select the most relevent landmarks. These strategies will have to take into account obstacles that represent a danger of collision and landmarks that yield a good localization.

\section{REFERENCES}

[1] Kai O. Arras, Jan Persson, Nicola Tomatis, and Roland Siegwart. Real-time obstacle avoidance for polygonal robots with a reduced dyna;ic window. In International Conference on Robotics and Automation, pages 3050-3055, Washington D.C, May 2002. IEEE.

[2] D. Kortenkampf and T. Weymouth. Topological Mapping for Mobile Robots using a Combination of Sonar and Vision Sensing. In National Conf. on Artificial Intelligence (AAAI), 1994.

[3] F. Lamiraux and D. Bonnafous. Reactive trajectory deformation for nonholonomic systems: Application to mobile robots. In International Conference on Robotics and Automation, pages 3099-3104, Washington D.C., May 2002. IEEE.

[4] J.C. Latombe. Robot motion planning. Kluwer Academic Publishers, 1991.

[5] J.-P. Laumond, editor. Robot Motion Planning and Control. Number ISBN 3-540-76219-1 in Lectures Notes in Control and Information Sciences 229. Springer, 1998.

[6] Javier Minguez, Luis Montano, Thierry Siméon, and Rachid Alami. Global nearness diagram navigation (gnd). In International Conference on Robotics and Automation, pages 33-39, Seoul KR, May 2001. IEEE.

[7] Philippe Moutarlier and Raja Chatila. Incremental free-space modelling from uncertain data by an autonomous mobile robot. In IROS, pages 200-213, Osaka Japan, November 1991. IEEE/RSJ.

[8] S. Quinlan and O. Khatib. Elastic bands: Connecting path planning and control. In International Conference on Robotics and Automation, Atlanta, GA, 1993. IEEE.

[9] José Santos-Victor, Raquel Vassallo, and Hans Schneebeli. TopologicalMaps for Visual Navigation. In Int. Conf. on Vision System, pages 21-36, 1999.

[10] Erik Schulenburg, Thilo Weigel, and Alexander Kleiner. Selflocalization in dynamic environments based on laser and vision data. In International Conference on Intelligent Robots and Systems, pages 998-1004, Las Vegas, Nevada, October 2003. IEEE.

[11] Thierry Simeon, Juan Cortes, Anis Sahbani, and Jean-Paul Laumond. A general manipulation task planner. In WAFR, pages 293309, December 2002.

[12] T. Siméon, JP. Laumond, C. Van Geem, and J. Cortés. Computer aided motion: Move3d within molog. In International Conference on Robotics and Automation, pages 1494-1499, Seoul KR, May 2001. IEEE.

[13] Alessandro C. Victorino, Patrick Rives, and Jean-Jacques Borrelly. A relative motion estimation by combining laser measurement and sensor based control. In International Conference on Robotics and Automation, pages 3924-3929, Washington D.C, May 2002. IEEE.
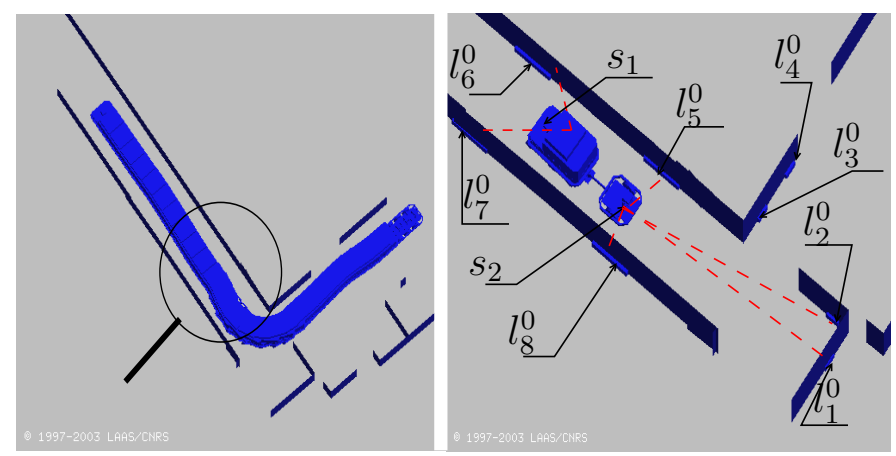

(a)
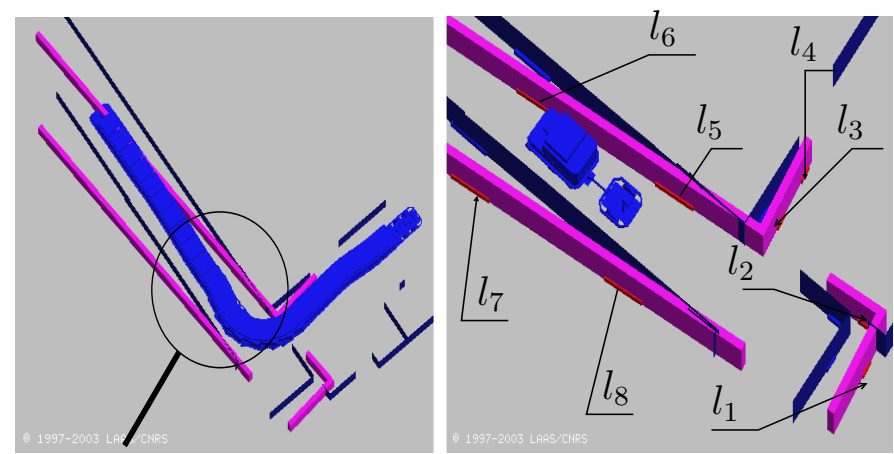

(b)
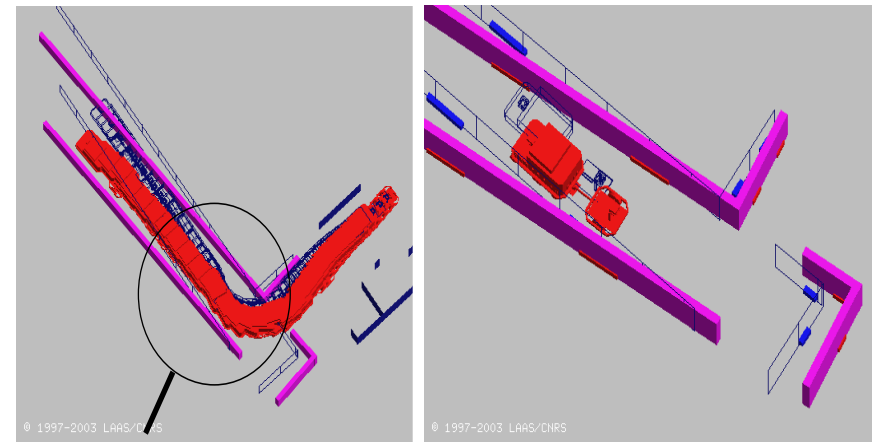

(c)

Fig. 5. Simulation Results. 